\title{
Systemic disorders in the pathophysiology of rheumatic heart disease
}

Mehmet Rami Helvaci (1)

Ibrahim Sari (2)

Abdulrazak Abyad (3)

Lesley Pocock (4)

(1) Specialist of Internal Medicine, MD

(2) Specialist of Cardiology, MD

(3) Middle-East Academy for Medicine of Aging, MD

(4) medi+WORLD International

Corresponding author:

Prof Dr Mehmet Rami Helvaci,

ALANYA, Antalya, Turkey

Phone: 00-90-506-4708759

Email:mramihelvaci@hotmail.com

Received September 2020. Accepted October 2020. Published November 1, 2020.

Please cite this article as: Helvaci MR et al. Systemic disorders in the pathophysiology of rheumatic heart disease. Middle East J Intern Med 2020; 13(2): 34-38 DOI: 10.5742/MEJIM2020.93785

\section{ABSTRACT}

Background: We tried to understand roles of systemic disorders in the pathophysiology of rheumatic heart disease (RHD).

Methods: All patients who applied to the Internal Medicine Units were included.

Results: We detected 29 females and 20 males with RHD. Their mean ages were 42.7 versus 33.0 years, respectively $(p=0.016)$. There were 26 cases with sickle cell diseases (SCDs), two cases with rheumatoid arthritis (RA), two cases with systemic lupus erythematosus (SLE), one case with juvenile rheumatoid arthritis (JRA), one case with common variable immunodeficiency, one case with celiac sprue, two cases with cirrhosis, and one case with chronic renal disease. In other words, there were associated systemic pathologies in $73.4 \%$ of RHD patients. Mitral valve was involved in $41.3 \%$, mitral and aortic valves were involved in $\mathbf{2 6 . 0 \%}$, mitral and tricuspid valves were involved in $17.3 \%$, aortic valve was involved in $8.6 \%$, and all of the three valves were involved in $6.5 \%$ of the cases. In another definition, mitral, aortic, and tricuspid valves were involved in $91.3 \%, 41.3 \%$, and $23.9 \%$ of cases, respectively. Interestingly, tricuspid valve was never involved alone, instead always together with mitral valve.
There were $69.3 \%$ of patients with mitral regurgitation, $26.5 \%$ with aortic regurgitation, $22.4 \%$ with tricuspid regurgitation, $20.4 \%$ with mitral stenosis, and $18.3 \%$ with aortic stenosis.

Conclusion: Systemic disorders including SCDs, RA, SLE, and JRA induce chronic inflammatory processes on vascular endothelium in the whole body, and terminate with an immunosuppression by several mechanisms that may be the cause of higher prevalence of RHD together with such disorders.

Key words: Rheumatic heart disease, sickle cell diseases, rheumatoid arthritis, systemic lupus erythematosus, chronic endothelial damage, atherosclerosis, immunosuppression 


\section{Introduction}

Chronic endothelial damage may be the major cause of aging by inducing disseminated tissue hypoxia all over the body. Much higher blood pressure (BP) of the afferent vasculature may be the major underlying cause, and probably whole afferent vasculature including capillaries are mainly involved in the process. Some of the well-known accelerators of the inflammatory process are physical inactivity, excess weight, smoking and alcohol for the development of irreversible consequences including obesity, hypertension, diabetes mellitus, cirrhosis, peripheric artery disease (PAD), chronic obstructive pulmonary disease (COPD), chronic renal disease (CRD), coronary heart disease (CHD), mesenteric ischemia, osteoporosis and stroke. They were researched under the title of metabolic syndrome in the literature, extensively (1-4). Similarly, sickle cell diseases (SCDs) are severe and chronic inflammatory processes on vascular endothelium, terminating with end-organ insufficiencies in early years of life. Hemoglobin S causes loss of elastic and biconcave disc shaped structures of red blood cells (RBCs). Probably loss of elasticity instead of shape is the main problem since sickling is rare in peripheric blood samples of the SCDs with associated thalassemia minor and human survival is not so affected in hereditary spherocytosis or elliptocytosis. Loss of elasticity is present during the whole lifespan, but exaggerated with various stresses of the body. The hard RBCs induced severe and chronic vascular endothelial damage, inflammation, edema, and fibrosis terminate with tissue hypoxia all over the body $(5,6)$. Capillary systems may mainly be involved in the process due to their distribution function for the hard bodies. In another definition, metabolic syndrome is an accelerated atherosclerotic process, and SCDs are an accelerated metabolic syndrome. We tried to understand roles of systemic disorders in the pathophysiology of rheumatic heart disease (RHD).

\section{Material and Methods}

The study was performed in Internal Medicine Units of the Dumlupinar and Mustafa Kemal Universities between August 2005 and July 2016. All patients applied to the Internal Medicine Units were included into the study. The SCDs were diagnosed with hemoglobin electrophoresis performed via high performance liquid chromatography. A complete physical examination of all study cases was performed by the same internist. A check up procedure including fasting plasma glucose, creatinine, hepatic function tests, markers of hepatitis viruses $\mathrm{A}, \mathrm{B}, \mathrm{C}$ and human immunodeficiency virus, a posterior-anterior chest $\mathrm{x}$ ray film, an electrocardiogram, a Doppler echocardiogram both to evaluate cardiac walls and valves and to measure systolic BP of pulmonary artery, and an abdominal ultrasonography were performed for all cases. CRD is diagnosed with a persistent serum creatinine level of $1.3 \mathrm{mg} / \mathrm{dL}$ in males and $1.2 \mathrm{mg} / \mathrm{dL}$ in females. Cirrhosis is diagnosed with physical examination, liver function tests, ultrasonographic evaluation, and tissue samples in case of requirement. RHD is diagnosed with the echocardiographic findings. Patients with rheumatoid arthritis (RA) are classified with the criteria of early rheumatoid arthritis (ERA) (7). The ERA criteria include a morning stiffness of 30 minutes or longer, arthritis of three or more joint areas, arthritis of hand joints, positivity of rheumatoid factor (RF), and positivity of anti-cyclic citrullinated peptide antibody (anti-CCP). RA is defined by the presence of three or more of the criteria. Systemic lupus erythematosus (SLE) is classified with the criteria of American College of Rheumatology of 1997 including discoid rash, immunologic features, photosensitivity, neurologic disorders (headache, depression, seizures, and psychosis), oral ulcers, malar rash, arthritis, serositis, hematologic disorders (leukopenia, lymphopenia, thrombocytopenia, and hemolytic anemia), antinuclear antibodies (ANA), and renal involvement (proteinuria, low complement, red blood cell casts, granular casts) (8). Four of them are required for classification but not for diagnosis. Juvenile rheumatoid arthritis (JRA) is classified, clinically (9). Common variable immunodeficiency (CVID) is diagnosed with the clinical history, serum immunoglobulin (Ig) levels, and exclusion of other possible causes (10). Celiac sprue is diagnosed by the clinical history, IgA antiendomysial antibodies, and jejunal biopsy in case of requirement (11). Eventually, all cases with the RHD and their associated disorders were detected. Independent-Samples t test was used as the method of statistical analysis.

\section{Results}

We detected 29 females and 20 males with the RHD. Their mean ages were $42.7 \pm 17.8(19-77)$ versus $33.0 \pm 9.1(22-55)$ years, respectively $(p=0.016)$. There were 26 cases with the SCDs, two cases with RA, two cases with SLE, one case with JRA, one case with CVID, one case with celiac sprue, two cases with cirrhosis, and one case with CRD. In other words, there were associated systemic pathologies in $73.4 \%$ of the RHD patients. Mitral valve alone was involved in $41.3 \%$, mitral and aortic valves were involved in $26.0 \%$, mitral and tricuspid valves were involved in $17.3 \%$, aortic valve alone was involved in $8.6 \%$, and all of the three valves were involved in $6.5 \%$ of the cases. In another definition, mitral, aortic, and tricuspid valves were involved in $91.3 \%, 41.3 \%$, and $23.9 \%$ of cases, respectively. Interestingly, tricuspid valve was never involved alone, instead always together with mitral valve. There were $69.3 \%$ of patients with mitral regurgitation, $26.5 \%$ with aortic regurgitation, $22.4 \%$ with tricuspid regurgitation, $20.4 \%$ with mitral stenosis, and $18.3 \%$ with aortic stenosis.

\section{Discussion}

Chronic endothelial damage may be the leading cause of aging in human beings. It may be the most common type of vasculitis all over the world at the moment. Whole afferent vasculature including capillaries may chiefly be involved in the process. Much higher BP of the afferent vasculature may be the major underlying cause by inducing recurrent injuries on endothelium. Therefore the term of venosclerosis is not as famous as atherosclerosis in the medical literature. Physical inactivity, excess weight, smoking, alcohol, chronic inflammations, prolonged infections, and cancers probably accelerate the process. Secondary to the chronic endothelial damage, inflammation, edema, and fibrosis, vascular walls become thickened, their lumens are narrowed, and they lose their elastic nature which reduces blood flow and increases systolic BP further. Although early withdrawal of underlying factors may delay terminal consequences, after development of cirrhosis, COPD, CRD, CHD, PAD, or stroke, endothelial changes cannot be reversed completely due to their fibrotic nature (12). 
SCDs are life-threatening hereditary disorders affecting around 100,000 individuals in the United States (13). As a difference from other causes of chronic endothelial damage, the SCDs may keep vascular endothelium particularly at the capillary level (14), because the capillary system is the main distributor of the hard RBCs into the tissues. The hard cells induced severe and chronic endothelial damage, inflammation, edema, and fibrosis terminate with end-organ insufficiencies in early years of life. As a result, mean lifespans of the patients were 48 years in females and 42 years in males in the literature (15), whereas they were 33.3 and 30.8 years, respectively in our previous study (16). Unfortunately, the great differences may be secondary to delayed diagnosis, delayed initiation of hydroxyurea therapy, and inadequate RBCs supports during medical and surgical emergencies in Antakya region of Turkey. Actually, RBCs supports must be given in all medical and surgical emergencies in which there is evidence of clinical deterioration in the SCDs $(17,18)$. RBCs supports decrease sickle cell concentration in the circulation, and suppress bone marrow for the production of abnormal RBCs. So it decreases sickling induced endothelial damage all over the body. According to our 20-year experiences, simple RBCs transfusions are superior to the exchange. First of all, preparation of one or two units of RBCs suspensions in each time rather than preparation of six units or more provides time for clinicians to prepare more units by preventing sudden death of such patients. Secondly, transfusion of one or two units of RBCs suspensions in each time decreases the severity of pain, and relaxes anxiety of the patients and surroundings in a short period of time. Thirdly, transfusions of lesser units of RBCs suspensions in each time decrease transfusion-related complications in the future. Fourthly, transfusions of RBCs suspensions in the secondary health centers prevent some deaths developed during transport to the tertiary centers for the exchange. Fifthly, transfusions of RBCs suspensions in the secondary health centers prevent some extra costs on the health system developed during the exchange in the tertiary centers. On the other hand, longer survival of females in the SCDs (15) and longer overall survival of females in the world (19) cannot be explained by the atherosclerotic effects of smoking and alcohol alone, instead it may be explained by stronger physical efforts of male sex in life that may terminate with an exaggerated sickling and an exaggerated chronic endothelial damage in their bodies (20).

RHD is caused by an autoimmune reaction against Group A $\beta$-hemolytic streptococci. The majority of morbidity and mortality associated with rheumatic fever is caused by its destructive effects on cardiac valves. It is characterized by repeated inflammation with fibrinous repair. Fibrosis and scarring of valve leaflets, commissures, and cusps leads to abnormalities that can result in valvular stenosis or regurgitation. The valvular endothelium is a prominent site of lymphocyte-induced damage. Normally, T cell activation is triggered by presentation of the bacterial antigens. In RHD, molecular mimicry results in incorrect $\mathrm{T}$ cell activation, and these $\mathrm{T}$ lymphocytes can go on to activate B cells, which will start to produce selfantigen-specific antibodies. This leads to an immune response attack mounted against tissues in the heart that are misidentified as pathogens. RHD usually occurs after repeated attacks. Rheumatic fever primarily affects children between the ages of 5 and 17 years. In one third of cases, the underlying Strepto- coccal infection develops without any symptom. On the other hand, some patients develop significant carditis that manifests as congestive heart failure. Unlike typical heart failure, rheumatic heart failure responds well to corticosteroids, probably due to its autoimmune nature. In Western countries, rheumatic fever has become fairly rare, probably due to the widespread use of antibiotics. RHD disproportionately affects women of reproductive age (21). Similarly, it was detected with a female ratio of $59.1 \%$, and the mean age of females was 42.7 years in the present study. Rheumatic tricuspid valve dysfunction is the rarest of all valvular diseases, and is often associated with leftsided valvular diseases (22). The prevalence of rheumatic tricuspid dysfunction was $8.4 \%$ in a previous study (23). In another study, rheumatic tricuspid valve disease was detected with a ratio of $7.7 \%$, and associated mitral valve disease was present in $99.3 \%$ of them (23). Whereas tricuspid valve was involved in $23.9 \%$ of cases, and never alone instead always together with mitral valve involvement in the present study. Mitral valve is involved in $97 \%$ of cases with the RHD (24), and mitral stenosis is classically caused by it (25). Similarly, mitral and aortic valves were involved in $91.3 \%$ and $41.3 \%$ of cases, respectively in the present study.

SCDs are severe inflammatory processes terminating with end-organ insufficiencies in early years of life (26). First of all, the SCDs are chronic hemolytic anemias in which the normal lifespan of RBCs decreased from the normal 120 to 15-25 days. Secondly, the severe and chronic endothelial inflammation all over the body causes an overlapping chronic disease anemia. Thirdly, the chronic hemolytic process may even cause folate and vitamin B12 deficiencies. Furthermore, end-organ insufficiencies can suppress the immune system of the patients. Frequent acute sinusitis, tonsillitis, and urinary tract infections are the common causes of painful crises and hospitalizations, and they can rapidly progress into the severe and life-threatening infections including pneumonia, meningitis, and sepsis due to the moderate to severe immunosuppression in such patients (27). For example, tonsillary hypertrophy is a common physical examination finding that may be the result of a prolonged infectious process due to the moderate to severe immunosuppression in them (28). Severe and prolonged endothelial inflammation induced prominent weight loss and cachexia are also frequent findings in the SCDs (6). As a result, menarch is retarded in females with the SCDs (29). Moderate to severe anemias, autosplenectomy, frequent painful crises, hospitalizations, invasive procedures, RBCs supports, medications, loss of appetite, cachexia, prevented normal daily activities, and a suppressed mood of the body may just be some of the possible reasons of immunosuppression in the SCDs (30-32). As a result, the significantly higher prevalence of RHD due to repeated bacterial infections should not be an amazing finding in patients with the SCDs.

RA is characterized by synovial inflammation and hypertrophy of the peripheral joints, potentially terminating with progressive destruction of articular and periarticular tissues (33). It typically affects small joints of hands and feet, but it can also affect larger joints (34). Fever, subcutaneous and visceral nodules, pleural and pericardial effusions, lymphadenopathy, splenomegaly, cytopenias, and episcleritis are just some samples of the extra-articular manifestations. Diagnosis is based 
on the clinical history, acute phase reactants, and autoantibodies including RF and anti-CCP (35). The presence of clinical or subclinical synovitis detected with ultrasonography or magnetic resonance imaging is essential for early diagnosis. RA may also present with a large joint monoarthritis or oligoarthritis. In cases presenting with monoarthritis, careful assessment for differential diagnosis is needed, particularly in the older individuals where other conditions such as gout, calcium pyrophosphate deposition disease, and osteoarthritis are more common (36). Early referral of patients with suspected synovitis, particularly in small joints of hands and feet, is important in long-term outcomes (37). On the other hand, RA may mimic many systemic disorders, particularly in young and middle-aged females due to the extra-articular manifestations. According to our experiences, the diagnosis of RA requires trained clinicians who are able to differentiate early symptoms of RA from other pathologies, particularly from SLE. SLE is an autoimmune disease, too, and mostly seen in women with a younger mean age (38). Although the similar clinical presentation types, similar treatment agents, and similar prognosis, RA may have a lower prevalence in society $(2.7 \%$ versus $6.0 \%$, $\mathrm{p}<0.001)$, similar prevalences in both genders $(50.0 \%$ versus $92.3 \%$ in females, $\mathrm{p}<0.001)$, and higher mean age of onset $(44.5$ versus 37.0 years, $p=0.038$ ) than the SLE (39). SLE can be distinguished by the characteristic skin lesions on light-exposed areas, oral aphthous lesions, nonerosive arthritis, positive antibodies to double-stranded deoxyribonucleic acid (DNA), renal and central nervous system (CNS) involvements, and cytopenias (38). Especially clinicians in the Hematology Clinics should be aware of SLE due to the associated thrombocytopenia in differential diagnosis with idiopathic thrombocytopenic purpura. The sera of most patients contain ANA, often including anti-double-stranded DNA antibodies (40). Articular symptoms are seen in $90 \%$ of patients, and they may exist for years before the diagnosis (41). For example, the average time from the onset of symptoms to diagnosis was 5 years in the above study (38). As a difference from RA, most lupus polyarthritis is nondestructive in nature. Cutaneous lesions include characteristic malar butterfly erythema, discoid lesions, and erythematous, firm, maculopapular lesions of face, sun-exposed areas of neck, upper chest, and elbows. Photosensitivity is seen in $40 \%$ of cases. Generalized lymphadenopathy is also common. CNS involvement may cause personality changes, stroke, epilepsy, and psychoses (42). Although the renal involvement may be fatal, the most common manifestation is proteinuria (43). There were increases in the incidences of renal involvement and neurological symptoms throughout the disease course (38). Earlystage SLE can be difficult to differentiate from RA if arthritic symptoms predominate (33-35). Thus, ANA and anti-doublestranded DNA antibodies should be studied in all patients suspected with RA $(36,37)$. Similarly, JRA is the most commonly seen chronic arthropathy before the age of 16 years (9). It is an autoimmune disorder, too. It can cause fever and anemia, and can also affect the heart, lungs, eyes, and the nervous system (9). The main difference between JRA and RA is that some children with JRA can get complete cure, while adults with RA usually have lifelong sysmptoms. Additionally, JRA may affect bone development as well as the child's growth. Beside that RF positivity is higher with RA than the JRA. The presence of RF positivity in the JRA indicates a higher risk of development of RA in adulthood. Immunosuppressive drugs, mainly the corti- costeroids and methotrexate, are the major treatment approaches for all of the three disorders. On the other hand, chronic disease anemia, frequent hospitalizations, invasive procedures, RBCs supports, medications, loss of appetite, cachexia, prevented normal daily activities, and a suppressed mood of the body may just be some of the possible reasons of immunosuppression with such disorders. As a result, the higher prevalence of RHD due to repeated bacterial infections should not be an amazing finding in them, too.

As a conclusion, systemic disorders including SCDs, RA, SLE, and JRA induce chronic inflammatory processes on vascular endothelium all over the body, and terminate with an immunosuppression by several mechanisms that may be the cause of higher prevalence of RHD together with such disorders.

\section{References}

1. Eckel RH, Grundy SM, Zimmet PZ. The metabolic syndrome. Lancet 2005; 365(9468): 1415-1428.

2. Helvaci MR, Kaya H, Sevinc A, Camci C. Body weight and white coat hypertension. Pak J Med Sci 2009; 25(6): 916-921.

3. Helvaci MR, Tonyali O, Abyad A, Pocock L. Smoking may be a cause of hypertriglyceridemia. World Family Med 2019; 17(8): 14-18.

4. Helvaci MR, Abyad A, Pocock L. Smoking-induced endothelial damage may increase plasma triglycerides. World Family Med 2019; 17(9): 37-42.

5. Helvaci MR, Gokce C, Davran R, Akkucuk S, Ugur M, Oruc C. Mortal quintet of sickle cell diseases. Int J Clin Exp Med 2015; 8(7): 11442-11448.

6. Helvaci MR, Kaya H. Effect of sickle cell diseases on height and weight. Pak J Med Sci 2011; 27(2): 361-364.

7. Zhao J, Su Y, Li R, Ye H, Zou Q, Fang Y, et al. Classification criteria of early rheumatoid arthritis and validation of its performance in a multi-centre cohort. Clin Exp Rheumatol 2014; 32(5): 667-673.

8 .Hochberg MC. Updating the American College of Rheumatology revised criteria for the classification of systemic lupus erythematosus. Arthritis Rheum 1997; 4D(9): 1725.

9. Aggarwal R, Ringold S, Khanna D, Neogi T, Johnson SR, Miller A, et al. Distinctions between diagnostic and classification criteria? Arthritis Care Res (Hoboken) 2015; 67(7): 891897.

10. Chapel H, Cunningham-Rundles C. Update in understanding common variable immunodeficiency disorders (CVIDs) and the management of patients with these conditions. $\mathrm{Br} \mathrm{J}$ Haematol 2009; 145(6): 709-727.

11. Vitoria JC, Arrieta A, Arranz C, Ayesta A, Sojo A, Maruri $\mathrm{N}$, et al. Antibodies to gliadin, endomysium, and tissue transglutaminase for the diagnosis of celiac disease. J Pediatr Gastroenterol Nutr 1999; 29: 571-574.

12. Helvaci MR, Aydin LY, Aydin Y. Digital clubbing may be an indicator of systemic atherosclerosis even at microvascular level. HealthMED 2012; 6(12): 3977-3981. 
13. Yawn BP, Buchanan GR, Afenyi-Annan AN, Ballas SK, Hassell KL, James AH, et al. Management of sickle cell disease: summary of the 2014 evidence-based report by expert panel members. JAMA 2014; 312(10): 1033-1048.

14. Helvaci MR, Aydin Y, Ayyildiz O. Hydroxyurea may prolong survival of sickle cell patients by decreasing frequency of painful crises. HealthMED 2013; 7(8): 2327-2332.

15. Platt OS, Brambilla DJ, Rosse WF, Milner PF, Castro $\mathrm{O}$, Steinberg $\mathrm{MH}$, et al. Mortality in sickle cell disease. Life expectancy and risk factors for early death. N Engl J Med 1994; 330(23): 1639-1644.

16. Helvaci MR, Davran R, Abyad A, Pocock L. What a high prevalence of rheumatic heart disease in sickle cell patients. World Family Med (in press).

17. Charache S, Scott JC, Charache P. "Acute chest syndrome" in adults with sickle cell anemia. Microbiology, treatment, and prevention. Arch Intern Med 1979; 139(1): 67-69.

18. Davies SC, Luce PJ, Win AA, Riordan JF, Brozovic M. Acute chest syndrome in sickle-cell disease. Lancet 1984; 1(8367): 3638.

19. Mathers CD, Sadana R, Salomon JA, Murray CJ, Lopez AD. Healthy life expectancy in 191 countries, 1999. Lancet 2001; 357(9269): 1685-1691.

20. Helvaci MR, Ayyildiz O, Gundogdu M. Gender differences in severity of sickle cell diseases in non-smokers. Pak J Med Sci 2013; 29(4): 1050-1054.

21. Chang AY, Nabbaale J, Okello E, Ssinabulya I, Barry M, Beaton AZ, et al. Outcomes and care quality metrics for women of reproductive age living with rheumatic heart disease in Uganda. J Am Heart Assoc 2020 Apr 21; 9(8): e015562.

22. Ben Ameur Y, Zairi I, Kamoun K, Ben Sassi M, Longo S, Battikh K, et al. Tricuspid dysfunction as a result of rheumatic disease. Tunis Med 2010; 88(11): 820-828.

23. Sultan FA, Moustafa SE, Tajik J, Warsame T, Emani $\mathrm{U}$, Alharthi $\mathrm{M}$, et al. Rheumatic tricuspid valve disease: an evidence-based systematic overview. J Heart Valve Dis 2010; 19(3): 374-382.

24. Kanagasingam A, Francis GR, Komagarajah B, Ladchumanan D, Sivapramyan A, Packiyarajah P, et al. Pattern of rheumatic valvular involvement and its contribution towards valvular malfunction in young adults. Ceylon Med J 2019; 64(3): 91-97. 25. Pressman GS, Ranjan R, Park DH, Shim CY, Hong GR. Degenerative mitral stenosis versus rheumatic mitral stenosis. Am J Cardiol 2020 Mar 5. pii: S0002-9149(20)30190-9

26. Helvaci MR, Yaprak M, Abyad A, Pocock L. Atherosclerotic background of hepatosteatosis in sickle cell diseases. World Family Med 2018; 16(3): 12-18.

27. Helvaci MR, Acipayam C, Aydogan A, Akkucuk S, Oruc C, Gokce C. Acute chest syndrome in severity of sickle cell diseases. Int J Clin Exp Med 2014; 7(12): 5790-5795.

28. Helvaci MR, Gokce C, Davran R, Acipayam C, Akkucuk S, Ugur M. Tonsilectomy in sickle cell diseases. Int J Clin Exp Med 2015; 8(3): 4586-4590.

29. Helvaci MR, Davarci M, Inci M, Yaprak M, Abyad A, Pocock L. Chronic endothelial inflammation and priapism in sickle cell diseases. World Family Med 2018; 16(4): 6-11.

30. Helvaci MR, Acipayam C, Davran R. Autosplenectomy in severity of sickle cell diseases. Int J Clin Exp Med 2014; 7(5): 1404-1409.
31. Helvaci MR, Aydogan A, Akkucuk S, Oruc C, Ugur M. Sickle cell diseases and ileus. Int J Clin Exp Med 2014; 7(9): 2871-2876.

32. Helvaci MR, Albayrak M, Balcik OS, Celebi H, Abyad A, Pocock L. Moderate iron deficiency anemia in the treatment of metabolic syndrome. Middle East J Intern Med 2017; 10 (3): 23-26.

33. Aletaha D, Neogi T, Silman AJ, Funovits J, Felson DT, Bingham CO 3rd, et al. 2010. Rheumatoid arthritis classification criteria: an American College of Rheumatology/European League Against Rheumatism collaborative initiative. Arthritis Rheum 2010; 62(9): 2569-2581.

34. Kourilovitch M, Galarza-Maldonado C, Ortiz-Prado E. Diagnosis and classification of rheumatoid arthritis. JAutoimmun 2014; 48-49: 26-30.

35. Sun J, Zhang Y, Liu L, Liu G. Diagnostic accuracy of combined tests of anti cyclic citrullinated peptide antibody and rheumatoid factor for rheumatoid arthritis: a meta-analysis. Clin Exp Rheumatol 2014; 32(1): 11-21.

36. Smolen JS, Aletaha D, Barton A, Burmester GR, Emery P, Firestein GS, et al. Rheumatoid arthritis. Nat Rev Dis Primers 2018; 4: 18001 .

37. Evans J, Negoescu A. Early referral improves long-term outcomes in rheumatoid arthritis. Practitioner 2017; 261(1804): 21-25.

38. Tomczyk-Socha M, Sikorska-Szaflik H, Frankowski M, Andrzejewska K, Odziomek A, Szmyrka M. Clinical and immunological characteristics of Polish patients with systemic lupus erythematosus. Adv Clin Exp Med 2018; 27(1): 57-61.

39. Helvaci MR, Tonyali O, Yaprak M, Abyad A, Pocock L. Rheumatoid arthritis may be one step further of systemic lupus erythematosus. World Family Med 2019; 17(4): 17-20.

40. Rovenský J, Tuchynová A. Systemic lupus erythematosus in the elderly. Autoimmun Rev 2008; 7(3): 235-239.

41. Yu C, Gershwin ME, Chang C. Diagnostic criteria for systemic lupus erythematosus: a critical review. J Autoimmun 2014; 48-49: 10-13.

42. Ichinose K. Unmet needs in systemic lupus erythematosus. Nihon Rinsho Meneki Gakkai Kaishi 2017; 40(6): 396-407. 43. Jaryal A, Vikrant S. Current status of lupus nephritis. Indian J Med Res 2017; 145(2): 167-178. 